\title{
Study on Cooperative Mechanism of Prefabricated Producers Based on Evolutionary Game Theory
}

\author{
Tongyao Feng, ${ }^{1,2}$ Shuangliang Tai, ${ }^{1,2}$ Chengshuang Sun,, ${ }^{1,2}$ and Qingpeng Man ${ }^{1,2}$ \\ ${ }^{1}$ Key Lab of Structures Dynamic Behavior and Control of the Ministry of Education, Harbin Institute of Technology, \\ Harbin 150090, China \\ ${ }^{2}$ Key Lab of Smart Prevention and Mitigation of Civil Engineering Disasters of the Ministry of Industry and Information Technology, \\ Harbin Institute of Technology, Harbin 150090, China
}

Correspondence should be addressed to Chengshuang Sun; suncs@vip.163.com

Received 3 April 2017; Accepted 12 June 2017; Published 17 July 2017

Academic Editor: Marzio Pennisi

Copyright (C) 2017 Tongyao Feng et al. This is an open access article distributed under the Creative Commons Attribution License, which permits unrestricted use, distribution, and reproduction in any medium, provided the original work is properly cited.

Good cooperation mechanism is an important guarantee for the advancement of industrialization construction. To strengthen the partnership between producers, we analyze the behavior evolution trend of both parties using an evolutionary game theory. Based on the original model, the mechanism of coordination and cooperation between prefabricated producers is explained under the condition of punishment and incentive. The results indicate that stable evolutionary strategies exist under both cooperation and noncooperation, and the evolutionary results are influenced by the initial proportion of both decision-making processes. The government can support the production enterprises to establish a solid partnership through effective punishment and incentive mechanisms to reduce the initial cost in the supply chain of prefabricated construction, resulting in a win-win situation.

\section{Introduction}

The construction industry is experiencing a transformation of production mode. One of the most important development directions is construction industrialization, which is in a critical stage of transformation and upgrading [1]. The experience of industrialized countries shows that prefabricated construction is at the core of transformation in helping to improve building quality, speed up construction progress, emit less pollution, save energy, and protect the environment.

To establish efficient partner relationships, some experts have explored the methods to strengthen the cooperation mechanism between producers and suppliers. Considering the problem of excess competition among construction enterprises, Wang and Zhang [2] studied the motivations for the formation of enterprise groups, by analyzing the game process of the strategy, to develop larger and structural rational construction groups and realize the cooperation of different enterprises. Pan and Wang [3] used quantitative methods to show that a reasonable market supervision mechanism is an important way to solve the problems of incomplete construction information, monopoly, and rentseeking. It is important to achieve business cooperation and a healthy and stable development of the industry. Yang and PeiHua [4] analyzed the dynamic evolution factors affecting the cooperation or competition mechanism between producers and suppliers in the supply chain through evolutionary game theory, including the initial proportion of the game subject, distribution of benefits, initial investment cost, and discount factor. Eriksson and Lindgren [5] discussed the effect of the game population scale, value of the income parameter, structure of initial scale, and mutation rate on the evolutionary process after analyzing the mechanism of mutation on cooperation, using the prisoner's dilemma model. Based on the analysis of evolution path and the influencing factors of horizontal enterprises, Su et al. [6] find that the growth of clusters promotes cooperative network formation, especially among small and medium-sized enterprises. Strengthening the cooperative behavior in cluster enterprises is an important way to expand the competitive advantage of industrial clusters. Jin et al. [7] analyzed that the construction supply chain integration remains in a low level at present and the 
amount of Engineering Procurement Construction (EPC) enterprise is relatively less in reality.

However, it has been found that scholars often discuss the cooperation mechanism between producers and suppliers rather than the horizontal cooperation between producers or suppliers. Precisely, strengthening the cooperation between producers is one of the most important methods in developing the chain. The advantages include timely sharing of information and cost returns from profit space obtained through the benefits of scale. In addition, it is beneficial for prefabricated producers to take out excess profits to improve product quality and increase $\mathrm{R} \& \mathrm{D}$ investment. The cooperation mechanism between producers results in further mass promotion of products into the market and provides a solid foundation for industrialization. Therefore, this study is mainly based on the problem of the lacking cooperation, and it attempts to use the imitator dynamic model in evolutionary game theory to analyze the cooperation and competition behavior of construction partners [8].

\section{Methodology}

In the long-term process of product collaborative development, producers need to game with each other several times. They can enter cooperation in the form of a contract or enter a rival competition relationship. Producers are not in complete rational consciousness. They present strong or poor abilities in a rational sense, analysis, and accurate identification and judgment of behavior, which cannot result in the most optimal strategy at the beginning. Assuming that the relationship is independent, we can conclude the evolution of competition between any other producers by studying the game model of two prefabricated producers [9].

Evolutionary game theory, which originated from biology, was put forward by Maynard and other scholars in the 20th century and has been widely used in many fields [10]. The core concepts of the evolutionary game theory are Evolutionary Stable Strategy (ESS) and Replicator Dynamic. Wu and Xiong [8] consider that humans usually use trial-anderror method to achieve the game equilibrium that makes the study more meaningful. This study examines the ESS and the influence of the system evolution caused by penalties, subsidies, and the cost of cooperation under the circumstance of the mechanism.

In order to explore the influence of various parameters existing in the process of the game on the final ESS, the following basic explanations are given:

(1) Producers 1 and 2 are limited rational players, and their strategy selection space is (cooperation $(C)$, competition $(D))$.

(2) If the probability of adopting the strategy of "cooperation" between producers 1 and 2 is represented by $x$ and $y$, respectively, then the probability of "competition" is $(1-x)$ and $(1-y)$, respectively. The state of the system evolution can be represented by $(x, y)$ on available area $[0,1] \times[0,1]$.

(3) Producers 1 and 2 decide to adopt their own strategy based on the cost benefits. The information of the
TABLE 1: Payment matrix.

\begin{tabular}{lcc}
\hline Producer 1 & \multicolumn{2}{c}{ Producer 2 } \\
\hline$C$ & $\pi_{1}+a \Delta \pi-c_{1}$ & $D$ \\
\hline \multirow{2}{*}{$D$} & $\pi_{2}+(1-a) \Delta \pi-c_{2}$ & $\pi_{1}-c_{1}-f_{1}$ \\
& $\pi_{1}+E_{1}$ & $\pi_{2}+E_{2}$ \\
& $\pi_{2}-c_{2}-f_{2}$ & $\pi_{1}$ \\
\hline
\end{tabular}

dynamic decision-making process is incomplete for both sides of the game [11].

Based on the above basic points, the parameters and the relevant symbol conventions are shown in Symbol and Definition.

When producers choose to cooperate, both sides can gain not only the benefits under the condition of normal market competition, but also some extra income. However, they must pay a certain cost for cooperation, which includes the corresponding labor, contract, and other fees in the practice. If one partner breaches the contract and takes the competition strategy alone after reaching the cooperation agreement, then the betraying partner holding a competition strategy will take some expected speculative returns called free riding or betrayed income, while the committed partner will suffer greater losses [12]. If the two sides are in competition, then they can only benefit from normal market competition. The net return of subjects in the construction supply chain can be calculated, and the game payment matrix is shown in Table 1.

We use the universal scaling to describe the problem. The payoffs producer 1 receives are $R$ (reward for mutual cooperation), $P$ (punishment for mutual defection), $T$ (temptation to defect), and $S$ (suckers payoff). Then the profits can be expressed in the simple matrix with $R, S, T$, and $P$. Facing the problem of different costs on opponent's strategy, Tanimoto [13] developed new parameters, $D g=T-R$ and $D r=P-S$. However, some scholars proposed that when $D g=D r$, which means the value between choice of cooperation and competition is equal when facing different strategies, the situation is called Donor \& Recipient Game, and it will depend on the value of $R-P$. Therefore, Wang et al. [14] introduce the third set of universal scaling parameters, $D g^{\prime}=D g /(R-P)$ and $D r^{\prime}=D r /(R-P)$, for 2 by 2 games to overcome the limitations of predicting the equilibrium states with reciprocity mechanisms. We show the parameters in Table 2 with the presentation of new set of universal scaling.

\section{Analysis on Model Parameters}

Assuming that the expected benefits of producer 1 choosing "cooperation" and "competition" strategies are $\mu_{11}$ and $\mu_{12}$, respectively, the expected return for producer 1 when it takes the cooperative strategy can be calculated as shown in Table 2

$$
\mu_{11}=y\left(a \Delta \pi+f_{1}\right)-\left(c_{1}+f_{1}-\pi_{1}\right) .
$$

The expected return for competitive strategy is

$$
\mu_{12}=y E_{1}+\pi_{1} \text {. }
$$


TABLE 2: Payment matrix in universal scaling.

\begin{tabular}{lcc}
\hline Producer 1 & \multicolumn{2}{c}{ Producer 2 } \\
& $C$ & $D$ \\
\hline$C$ & $R=1$ & $S=-D r^{\prime}$ \\
$D$ & $T=1+D g^{\prime}$ & $P=0$ \\
\hline
\end{tabular}

The average expected return for producer 1 can be calculated as

$$
\overline{\mu_{1}}=x y\left(a \Delta \pi+f_{1}-E_{1}\right)-x\left(c_{1}+f_{1}\right)+y E_{1}+\pi_{1} .
$$

According to the Malthusian dynamic equation, the Reproduction Dynamic Equation (RDE) of producer 1 is

$$
\frac{d x}{d t}=x(1-x)\left[y\left(a \Delta \pi_{1}+f_{1}-E_{1}\right)-\left(c_{1}+f_{1}\right)\right] .
$$

The RDE of producer 2 is

$$
\frac{d y}{d t}=y(1-y)\left\{x\left[(1-a) \Delta \pi+f_{2}-E_{2}\right]-\left(c_{2}+f_{2}\right)\right\} \text {. }
$$

The replicated dynamic state can be obtained, respectively, by making simultaneous RDEs (4) and (5) equal to 0:

$$
\begin{aligned}
& x_{1}{ }^{*}=0, \\
& x_{2}{ }^{*}=1, \\
& y^{*}=\frac{\left(c_{1}+f_{1}\right)}{\left(a \Delta \pi+f_{1}-E_{1}\right)} .
\end{aligned}
$$

Similarly,

$$
\begin{aligned}
& y_{1}{ }^{*}=0, \\
& y_{2}{ }^{*}=1, \\
& x^{*}=\frac{\left(c_{2}+f_{2}\right)}{\left[(1-a) \Delta \pi+f_{2}-E_{2}\right]} .
\end{aligned}
$$

It can be seen that there are five local equilibrium points in the system, namely, $(0,0),(0,1),(1,0),(1,1)$, and $\left(x^{*}, y^{*}\right)$.

The stability of the dynamic system equilibrium point, described as the differential equations, can be obtained by analyzing the structure of the Jacobian matrix of the system according to Friedman's method [15]. The system's Jacobian matrix can be calculated as

$$
J=\left(\begin{array}{ll}
\frac{\partial(d x / d t)}{\partial x} & \frac{\partial(d x / d t)}{\partial y} \\
\frac{\partial(d y / d t)}{\partial x} & \frac{\partial(d y / d t)}{\partial y}
\end{array}\right)=\left(\begin{array}{ll}
a_{11} & a_{12} \\
a_{21} & a_{22}
\end{array}\right)
$$

where

$$
\begin{aligned}
& a_{11}=(1-2 x)\left[y\left(a \Delta \pi+f_{1}-E_{1}\right)-\left(c_{1}+f_{1}\right)\right], \\
& a_{12}=x(1-x)\left(a \Delta \pi+f_{1}-E_{1}\right), \\
& a_{21}=y(1-y)\left[(1-a) \Delta \pi+f_{2}-E_{2}\right], \\
& a_{22}=(1-2 y)\left\{x\left[(1-a) \Delta \pi+f_{2}-E_{2}\right]-\left(c_{2}+f_{2}\right)\right\} .
\end{aligned}
$$

If conditions are satisfied with the value of the balance point, $\operatorname{Tr}(J)=a_{11}+a_{22}<0$, $\operatorname{Det}(J)=a_{11} a_{22}-a_{12} a_{21}>0$, the equilibrium point can be considered to have local stability for the ESS at this point, as shown in Table 3.

When $a \Delta \pi-E_{1}>c_{1}$, the producer's betrayal income for free riding is less than the difference between extra income for cooperation and the cost of cooperation. It can be analyzed from the above Jacobian matrix and local stability results that five local equilibrium points for stability analysis have been obtained.

(1) If $0<y<\left(c_{1}+f_{1}\right) /\left(a \Delta \pi+f_{1}-E_{1}\right)$, then there is a stable equilibrium solution for RDE named $x=$ 0 after taking $x=0$ and $x=1$ into formula (9) and concluding $a_{11}<0$ and $a_{11}>0$, respectively. This means that when the probability of producers 1 and 2 choosing to cooperate with each other is less than $\left(c_{1}+f_{1}\right) /\left(a \Delta \pi+f_{1}-E_{1}\right)$, the producer 1 will tend to choose a competitive strategy to deal with the external environment after a long period of mutual effects in the cooperative system.

(2) If $\left(c_{1}+f_{1}\right) /\left(a \Delta \pi+f_{1}-E_{1}\right)<y<1$, then there is a stable equilibrium solution for RDE named $x=1$ after taking $x=0$ and $x=1$ into formula (9) and concluding $a_{11}>$ 0 and $a_{11}<0$, respectively, which means that when the probability of producer 2 choosing to cooperate is more than $\left(c_{1}+f_{1}\right) /\left(a \Delta \pi+f_{1}-E_{1}\right)$, producer 1 will tend to choose the same strategy to deal with the external environment after a long period of mutual evolution.

(3) If $y=\left(c_{1}+f_{1}\right) /\left(a \Delta \pi+f_{1}-E_{1}\right)$, which means the probability of cooperation is exactly $y=\left(c_{1}+f_{1}\right) /(a \Delta \pi+$ $f_{1}-E_{1}$ ), then $a_{11}=0$ can always be established. In Figure 1 , the broken line $A-D-B$ shows the boundary that it is not affected by external factors, indicating that there is a stable equilibrium solution, whether producer 1 selects cooperation or competition. The stable equilibrium is a pure strategy under the complete information, which is difficult to achieve in reality.

Similarly, we can take the perspective of producer 2 to get the same conclusion.

The dynamic trajectory of the system evolution is shown in Figure 1, and the final evolution result is determined by the initial state of the system and the evolutionary critical line $A$ $D-B$. From an intuitive point of view, the probability that the system will evolve towards absolute competition and absolute cooperation depends on the ratio of area $O-A-D-B$ to area $C$ $A-D-B$.

From the analysis of Table 3 and Figure 1, we can see that there are two pure strategy Nash equilibria in the game, $(C, C)$ and $(D, D)$. Obviously, it is consistent with the equilibrium of Stag Hunt games where bistable equilibria appear in case of symmetric 2 by 2 games [16, 17].

\section{Model Evolution Based on External Conditions}

4.1. Evolutionary Game Based on Punishment Mechanism. As can be seen from the third-part analysis, if one does not restrict or constrain the other party in the free market, the betrayed party may get returns from the free-ride behavior of unilateral agreement breach. After a certain period of time, 
TABLE 3: System equilibrium point stability analysis.

\begin{tabular}{lccc}
\hline Balance point & $\operatorname{Tr}(J)$ & Det $(J)$ & Local stability \\
\hline$(0,0)$ & - & + & ESS \\
$(0,1)$ & + & + & Unstable \\
$(1,0)$ & + & + & Unstable \\
$(1,1)$ & - & + & ESS \\
$\left(x^{*}, y^{*}\right)$ & 0 & - & Saddle point \\
\hline
\end{tabular}

The position of the saddle point represents the proportion of the initial decision of the game subject.

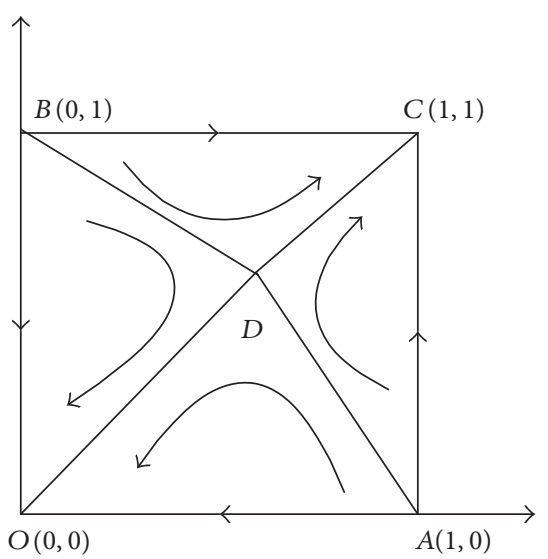

FIGURE 1: Dynamic phase of evolutionary game between prefabricated producers.

the party that keeps the commitment will be aware of the other party's strategy and choose to free ride more frequently in order to protect its own interests. From the general and long-term perspective, this approach is not conducive to the entire construction supply chain, as it does not foster a stable and healthy development.

In order to ensure that the supply chain collaborative product development cooperation between the two sides does not appear opportunistic and to ensure that cooperation is achieved in the end, it is necessary to introduce a punishment mechanism to restrict the occurrence of default. Therefore, in practice, assuming one party cooperates and the other does not, the noncooperative party will be punished and the penalty will be collected by the partner. We set the probability of default as $p$ and the penalty line as $Q$. This punishment can be specified in the agreement or it could be a government regulation. At this point, the game payment matrix based on the penalty mechanism is shown in Table 4 .

According to the Malthusian dynamic equation, the RDE of producer 1 is

$$
\begin{aligned}
& \frac{d x}{d t} \\
& \quad=x(1-x)\left[y\left(a \Delta \pi+f_{1}-E_{1}\right)-\left(c_{1}+f_{1}-p Q\right)\right] .
\end{aligned}
$$

The RDE of producer 2 is

$$
\begin{aligned}
& \frac{d y}{d t}=y(1-y) \\
& \quad \cdot\left\{x\left[(1-a) \Delta \pi+f_{2}-E_{2}\right]-\left(c_{2}+f_{2}-p Q\right)\right\} .
\end{aligned}
$$

TABLE 4: Payment matrix based on penalty mechanism.

\begin{tabular}{lcc}
\hline Producer 1 & \multicolumn{2}{c}{ Producer 2 } \\
\hline$C$ & $\pi_{1}+a \Delta \pi-c_{1}$ & $\pi_{1}-c_{1}-f_{1}+p Q$ \\
& $\pi_{2}+(1-a) \Delta \pi-c_{2}$ & $\pi_{2}+E_{2}-p Q$ \\
$D$ & $\pi_{1}+E_{1}-p Q$ & $\pi_{1}$ \\
& $\pi_{2}-c_{2}-f_{2}+p Q$ & $\pi_{2}$ \\
\hline
\end{tabular}

The system has four balance points named $O(0,0)$, $A(1,0), B(0,1)$, and $C(1,1)$. According to the stability theory, it can be seen that when the penalty mechanism is introduced, there exists only one evolutionary equilibrium point in the system $C(1,1)$, as long as $p Q>\max \left\{c_{1}+f_{1}, c_{2}+f_{2}\right\}$, which means the betrayal penalty is sufficient to compensate the loss of the betrayed partner. The point $C$ represents (cooperation, cooperation). Evolution phase is shown in Figure 2.

4.2. Evolutionary Game Based on Incentive Mechanism. One of the reasons why prefabricated construction development is slow in China is the cost of production and use. The prefabricated component producer at the front of the construction supply chain is not powerful enough to form a sufficiently strong industrial scale and create the basic conditions for the huge advancement. It is necessary for the government and enterprises to encourage one another to achieve the economic benefits of prefabricated construction. The government can guide producers to produce high-quality prefabricated components on large-scale through tax breaks or fiscal subsidies. Upstream and downstream enterprises can also make preferential consultations to achieve the completion of construction and reduce the unnecessary intermediate links, in order to maximize the economic benefits.

To encourage producers to reduce construction costs by strengthening cooperation, the main incentive subject can enhance the motivation and the expected level of income of enterprises through incentive mechanisms. Assuming that the government conducts monetary rewards for companies actively engaged in cooperation and the reward amount is $V$, the game payment matrix will be as shown in Table 5 .

According to the Malthusian dynamic equation, the RDE of producer 1 is

$$
\frac{d x}{d t}=x(1-x)\left[y\left(a \Delta \pi+f_{1}-E_{1}\right)-\left(c_{1}+f_{1}-V\right)\right] .
$$


TABLE 5: Payment matrix based on incentive mechanism.

\begin{tabular}{|c|c|c|}
\hline \multirow{2}{*}{ Producer 1} & \multicolumn{2}{|c|}{ Producer 2} \\
\hline & C & $D$ \\
\hline C & $\begin{array}{c}\pi_{1}+a \Delta \pi-c_{1}+V \\
\pi_{2}+(1-a) \Delta \pi-c_{2}+V\end{array}$ & $\begin{array}{c}\pi_{1}-c_{1}-f_{1}+p Q+V \\
\pi_{2}+E_{2}-p Q\end{array}$ \\
\hline$D$ & $\begin{array}{c}\pi_{1}+E_{1}-p Q \\
\pi_{2}-f_{1}+p O+V\end{array}$ & $\pi_{1}$ \\
\hline & $\pi_{2}-c_{2}-f_{2}+p Q+V$ & $\pi_{2}$ \\
\hline
\end{tabular}

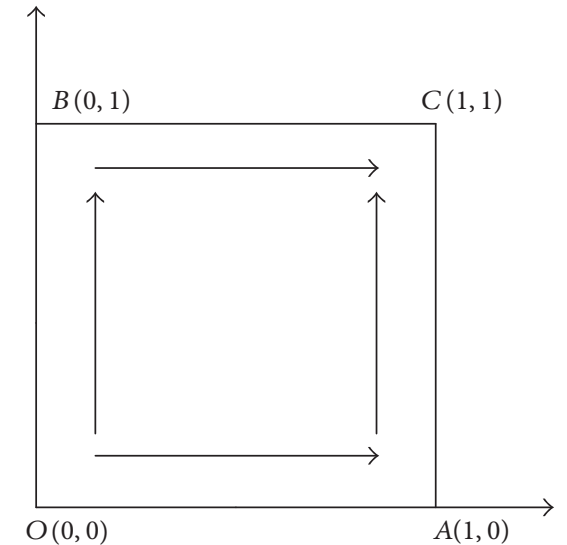

FIGURE 2: Dynamic phase of evolutionary game based on the penalty mechanism.

Similarly, the RDE of producer 2 is

$$
\begin{aligned}
\frac{d y}{d t}= & y(1-y) \\
& \cdot\left\{x\left[(1-a) \Delta \pi+f_{2}-E_{2}\right]-\left(c_{2}+f_{2}-V\right)\right\} .
\end{aligned}
$$

The system still has four balance points. According to the stability theory, it can be seen that when the incentive mechanism is introduced, there exists only one evolutionary equilibrium point $(1,1)$ in the system, as long as $V>\max \left\{c_{1}+\right.$ $\left.f_{1}, c_{2}+f_{2}\right\}$, which means the incentive amount is sufficient to compensate the loss of the partner. The point represents (cooperation, cooperation). This evolution phase is the same with Figure 2.

\section{Conclusion}

In this study, the evolutionary game method is used to establish the cooperation mechanism model among producers. Based on the analysis of the influencing factors on the long-term cooperation (including excess returns), ratio of excess returns, total cost of cooperation, and loss of unilateral competition, we further consider the impact on strategies selection on the basis of penalties and incentive mechanisms.

(1) When producer's betrayal income for free riding is less than the difference between extra income for cooperation and the cost of cooperation, there are two stable strategies, cooperation and noncooperation. The final evolution result is influenced by the ratio of the producer's initial choice.
(2) When the penalty mechanism is introduced, the betrayer will suffer greater loss because of the high amount of betrayal fines, and the betrayed partner will be motivated to continue to cooperate because of the betrayal fines they gain. Therefore, the results of both sides will be cooperation as long as the penalty is sufficient to compensate the loss of the partners.

(3) When the incentive mechanism is introduced, if the incentive amount can compensate for the cost of cooperation and the loss caused by the other party's betrayal, the producer will reduce the worries regarding betrayal. At the same time, a reasonable incentive also motivates enterprises to improve production.

(4) It can be seen from the position of the saddle point that the behavior decision of the game subject is influenced by the cooperative value-added income, the unilateral cooperation value-added income, the free-ride income, and the cooperation cost. When these factors are determined, the probability of the game subject decision is affected by others.

From the analysis and adjustment of the construction supply chain evolutionary game model, we can see that strengthening the cooperation between enterprises in the supply chain can be realized from the following two aspects:

(1) Reduce the cost of cooperation and the loss incurred by a partner during free riding by the other partner. For example, incentives must be given to positive cooperative producers, including important incentives such as material, monetary, and implicit incentives such as honors and encouragement. Besides, financial subsidies, policy concessions, and loan discounts are necessary to reduce the financial burden. Further, the punishment for malicious betrayal and taking it as partners' incentives significantly motivates the producer.

(2) Increase the extra benefits for cooperation, such as taking substantial incentives to increase the expected benefits of resources, environment, and economy and adjusting policies in a timely manner according to market operation and feedback. It is necessary to establish a favorable cooperation environment to improve the trust between enterprises and maintain a solid partnership to achieve a win-win situation.

The cooperation and competition relationship between prefabricated component producers is very complicated. This article assumes that producer 1 and producer 2 are independent individuals at present to explore their behavioral relationships. However, we know that sometimes producers may have interactions in reality, and their behavioral relationships are no longer independent completely. The research results provided by this paper cannot be inappropriate in this nonindependent situation. The nonindependent relations between prefabricated component producers are also very 
significant for practical application and would be left behind to further research.

\section{Symbol and Definition}

$\pi$ : The normal returns when the two sides do not cooperate

$\Delta \pi: \quad$ The excess returns when the two sides cooperate

a: The distribution coefficient of excess returns due to cooperation

c: The costs of the two sides pay for cooperation

$f$ : The loss to a partner when the other partner takes a competitive strategy while the first partner keeps commitment

E: The additional returns or betrayed income due to partners' technology spillover or their own absorptive capacity gained by the "free riding" of the noncooperative player

$\mu: \quad$ The expected return of the game subject $x, y$ : The percentage of group strategy selection.

\section{Conflicts of Interest}

The authors declare that there are no conflicts of interest regarding the publication of this paper.

\section{Acknowledgments}

The research was supported by the National "13th Five-Year" Key Research and Development Program of China under Grant no. 71071043.

\section{References}

[1] E. Bonamente, M. C. Merico, and S. Rinaldi, "Environmental impact of industrial prefabricated buildings: carbon and energy footprint analysis based on an LCA approach," Energy Procedia, vol. 61, pp. 2841-2844, 2014.

[2] Y. Wang and F.-L. Zhang, "Research on the construction enterprise group's formation mechanism based on evolutionary game," in Proceedings of the 4th International Conference on Computer Sciences and Convergence Information Technology (ICCIT '09), pp. 615-619, Seoul, South Korea, November 2009.

[3] H. Pan and X. Q. Wang, "Study on government regulation of construction enterprises based on evolutionary game theory," in Proceedings of the 18th International Conference on Industrial Engineering and Engineering Management, pp. 222-225, IEEE, Changchun, China, September 2011.

[4] P. Yang and F. Pei-Hua, "The cooperation and competition mechanism of supply chain based on evolutionary game theory," Lecture Notes in Electrical Engineering, vol. 107, pp. 12591265, 2012.

[5] A. Eriksson and K. Lindgren, "Cooperation driven by mutations in multi-person prisoner's dilemma," Journal of Theoretical Biology, vol. 232, no. 3, pp. 399-409, 2005.

[6] Y. Su, G. Chai, L. Gong, and Z. Li, "Evolutionary analysis on cooperative behavior of cluster enterprises in supply chain network," in Proceedings of the 4th International Conference on Business Intelligence and Financial Engineering (BIFE '11), pp. 575-579, Wuhan, China, October 2011.

[7] L. Jin, C. B. Liu, and X. J. Li, "Evolutionary game analysis of supply chain cooperation in China," Construction Economy, no. 6, pp. 71-73, 2009 (Chinese).

[8] X.-X. Wu and W.-Q. Xiong, "Evolutionary game analysis of the reverse supply chain based on the government subsidy mechanism," in Proceedings of the 2012 2nd International Conference on Business Computing and Global Informatization (BCGIN '12), pp. 99-102, Shanghai, China, October 2012.

[9] J. M. Allen and R. B. Hoyle, "Asynchronous updates can promote the evolution of cooperation on multiplex networks," Physica A: Statistical Mechanics and its Applications, vol. 471, pp. 607-619, 2017.

[10] C.-T. Zhang and L.-P. Liu, "Research on coordination mechanism in three-level green supply chain under non-cooperative game," Applied Mathematical Modelling. Simulation and Computation for Engineering and Environmental Systems, vol. 37, no. 5, pp. 3369-3379, 2013.

[11] E. Frey, "Evolutionary game theory: theoretical concepts and applications to microbial communities," Physica A. Statistical Mechanics and its Applications, vol. 389, no. 20, pp. 4265-4298, 2010.

[12] P. Ji, X. Ma, and G. Li, "Developing green purchasing relationships for the manufacturing industry: an evolutionary game theory perspective," International Journal of Production Economics, vol. 166, pp. 155-162, 2015.

[13] J. Tanimoto, "A simple scaling of the effectiveness of supporting mutual cooperation in donor-recipient games by various reciprocity mechanisms," BioSystems, vol. 96, no. 1, pp. 29-34, 2009.

[14] Z. Wang, S. Kokubo, M. Jusup, and J. Tanimoto, "Universal scaling for the dilemma strength in evolutionary games," Physics of Life Reviews, vol. 14, pp. 1-30, 2015.

[15] D. Friedman, "Evolutionary games in economics," Econometrica, vol. 59, no. 3, pp. 637-666, 1991.

[16] J. W. Weibull, Evolutionary Game Theory, The MIT Press, Cambridge, Mass, USA, 1997.

[17] J. Tanimoto, Fundamentals of Evolutionary Game Theory and Its Applications, vol. 6, Springer, Tokyo, Japan, 2015. 


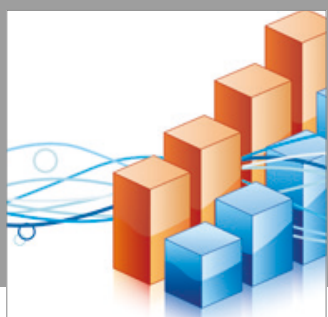

Advances in

Operations Research

vatersals

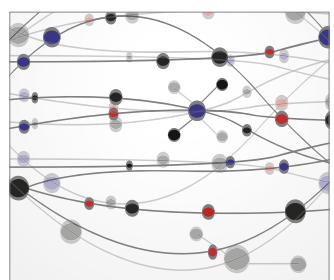

\section{The Scientific} World Journal
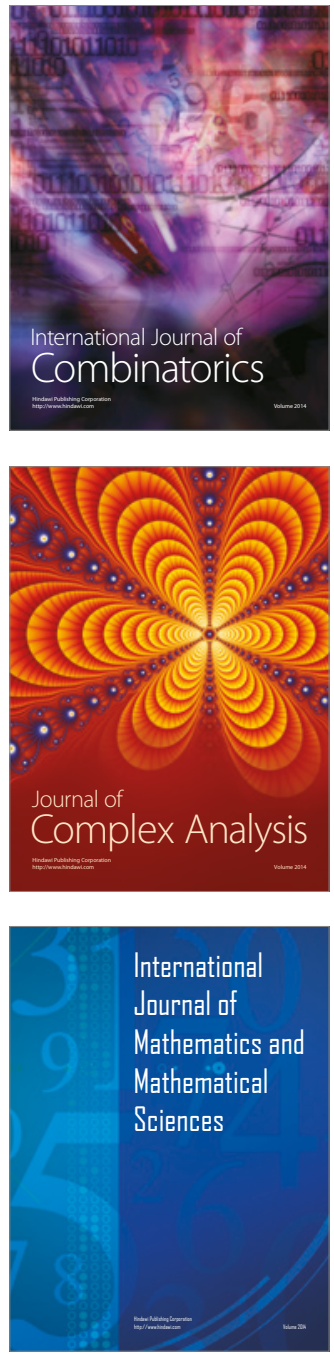
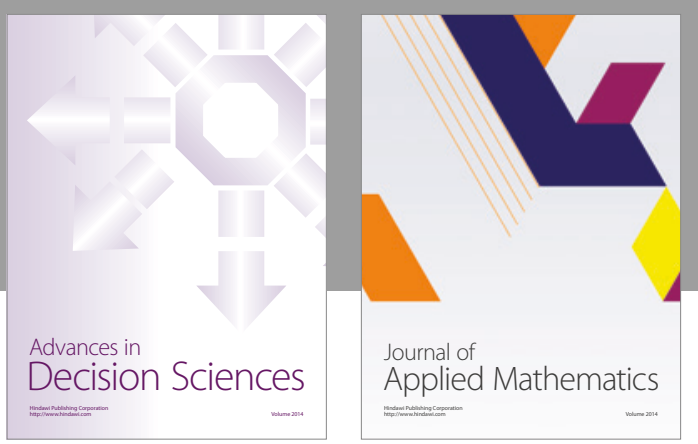

Algebra

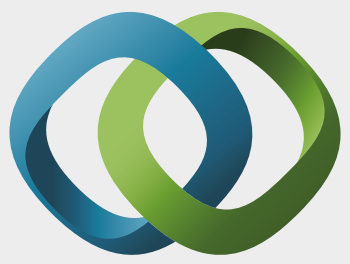

\section{Hindawi}

Submit your manuscripts at

https://www.hindawi.com
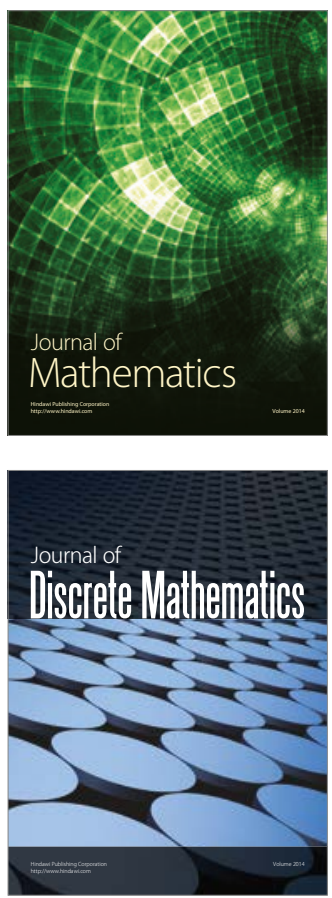

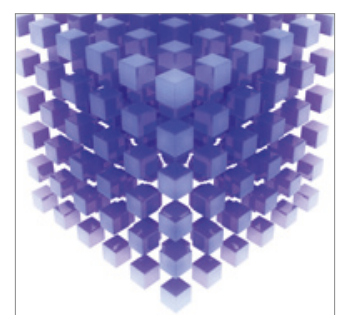

Mathematical Problems in Engineering
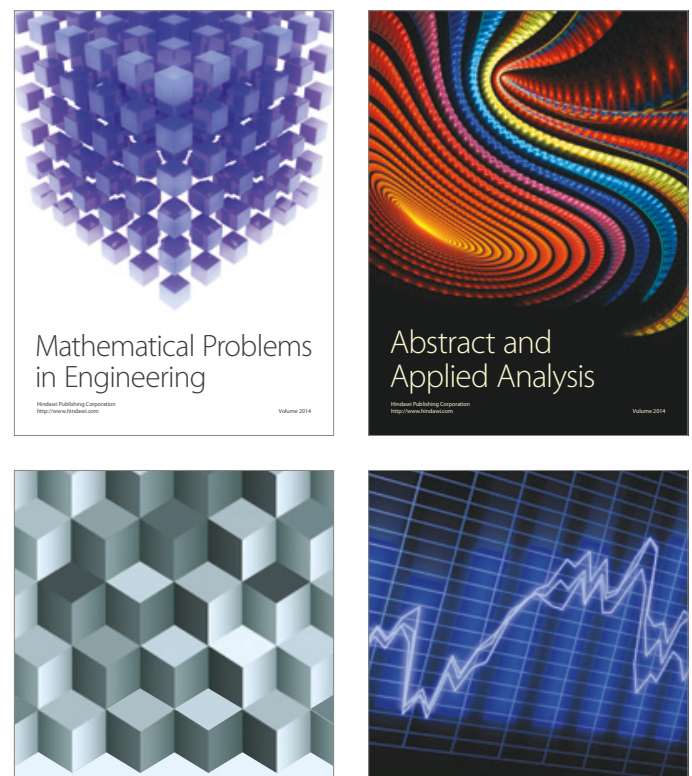

Journal of

Function Spaces

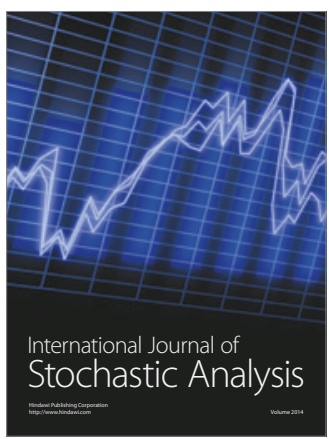

Probability and Statistics
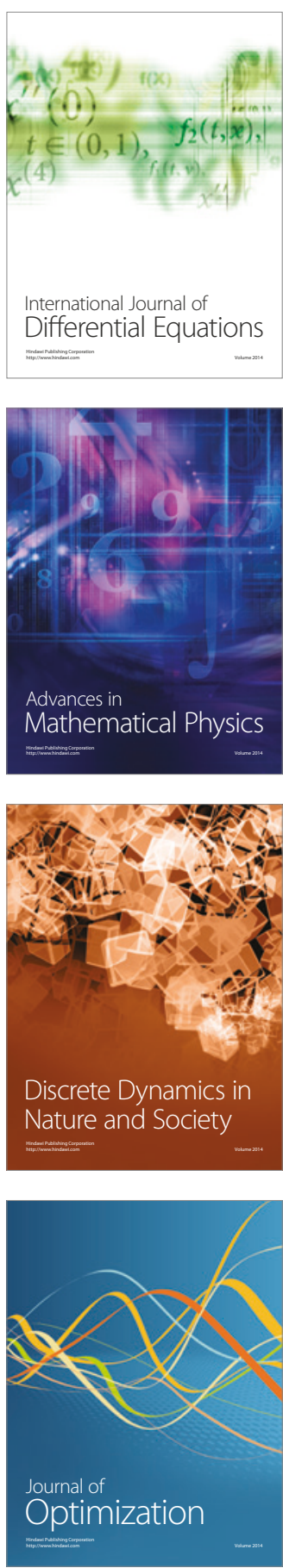